\title{
Tradução e Adaptação para o Português do Behavior Rating Inventory of Executive Function - BRIEF
}

\author{
Translation and Adaptation into Portuguese of the Behavior \\ Rating Inventory of Executive Function - BRIEF
}

\author{
Daniela de Bustamante Carim*, Monica C. Miranda \& Orlando F. Amodeo Bueno \\ Universidade Federal de São Paulo (UNIFESP), São Paulo, Brasil
}

\begin{abstract}
Resumo
O objetivo do presente estudo foi traduzir e adaptar, para o português, o BRIEF. O processo de tradução e adaptação seguiu os passos recomendados na literatura internacional. Além disso, foi realizada uma análise inicial das propriedades psicométricas. A versão final foi administrada numa amostra constituída por 277 pais e 282 professores de crianças entre 5 e 11 anos de idade, 112 adolescentes entre 11 e 18 anos da Cidade do Rio de Janeiro. Os valores dos Alfas de Cronbach oscilaram entre 0,901 e 0,957, considerando as 3 formas. A análise de componentes principais identificou duas dimensões. Os valores de adequada consistência interna encontrados na versão brasileira se assemelham aos da versão original, assegurando boa qualidade do processo de tradução e adaptação.

Palavras-chave: Função executiva, desenvolvimento, avaliação, adaptação transcultural.

Abstract

The aim of this study was to translate and adapt the BRIEF into Portuguese. The process of translation and adaptation followed the steps recommended in the international literature. Besides that, an initial analysis of psychometric properties was performed. The final version was administered in a sample of 277 parents, 282 teachers of children between 5 and 11 years old, and 112 adolescents between 11 and 18 years old from the city of Rio de Janeiro. The Cronbach Alphas ranged between .901 and .957 considering the three forms. The main component analysis extracted two dimensions. The internal consistency values found in the Brazilian version resembled the original version, ensuring good quality in the process of translation and adaptation.

Keywords: Executive function, development, evaluation, cultural adaptation.
\end{abstract}

Diversos autores têm discutido se a função executiva é um sistema unitário ou multidimensional (Bodnar, Prahme, Cutting, Denckla, \& Mahone, 2007; Gioia, Isquith, Kenworthy, \& Barton, 2002; Sullivan \& Riccio, 2007; Weyandt, 2005), mas parecem concordar que este termo é um construto para um conjunto de funções inter-relacionadas como capacidade de antecipar, planejar e organizar, iniciar ações, inibir interferências distratoras, monitorar o processo, alterar atividade quando necessário gerando uma nova ação (flexibilidade) e memória operacional.

Historicamente, a avaliação clínica das funções executivas tem sido um desafio dado sua essência dinâmica (Denckla, 1994). Até recentemente, neuropsicólogos confiavam principalmente nas avaliações laboratoriais das funções executivas como o Wisconsin Card Sorting Test (Heaton, Chelune, Talley, Kay, \& Curtiss, 1993). Entretanto, é frequentemente difícil extrapolar destas mensurações

"Endereço para correspondência: Rua Visconde de Pirajá, 547 sala 709, Ipanema, Rio de Janeiro, Brasil 2241-0002. E-mail: dcarim@uol.com.br para os comportamentos da vida real (Slick, Lautzenhiser, Sherman, \& Eyrl, 2006). Além do mais, a natureza estruturada de uma típica situação de avaliação freqüentemente não aloca domínios importantes da função executiva. Isto é, em diversas situações de avaliação, o examinador oferece a estrutura, a organização, direção e planejamento assim como pistas e monitoramento necessário para um bom desempenho do sujeito e, desse modo, o examinador funciona como um controle executivo externo (Gioia et al., 2002). Apesar das funções executivas serem cruciais para o funcionamento eficaz da criança na escola e na sociedade como um todo, há escassez de instrumentos apropriados para avaliar esse construto (Donders, 2002).

Estudiosos têm desenvolvido escalas ecológicas para a avaliação das funções executivas em crianças e adolescentes, dentre estas escalas, destaca-se o BRIEF ("Behavior Rating Inventory of Executive Function") desenvolvido por Gioia, Isquith, Guy e Kenworthy (2000). O BRIEF é um questionário para pais, professores e adolescentes que avalia o comportamento das funções executivas em casa e no ambiente escolar. É projetado para uma ampla gama 
de crianças e adolescentes, com idade entre 5 e 18 anos de idade. Há três formas do BRIEF: a pessoal é uma medida de auto-relato projetada para ser preenchida pelo próprio sujeito (com idades de 11 a 18 anos), constituída por 80 questões, que captura o ponto de vista do respondente acerca de sua percepção de funcionamento executivo no ambiente cotidiano. A forma para pais e professores, é constituída por questionários com 86 questões cada um, respondida pelos pais e professores de crianças e também de adolescentes, sendo a faixa compreendida para essas 2 formas entre 5 e 18 anos. Os respondentes são instruídos a graduar numa escala (nunca, algumas vezes, freqüen-temente) qual a freqüência que a criança apresenta o comportamento problema.

$O$ resultado da escala oferece uma mensuração global do funcionamento executivo assim como oferece dois índices: de regulação do comportamento e metacognição. $\mathrm{O}$ índice de regulação do comportamento inclui três domínios: inibição, flexibilidade e controle emocional; enquanto que o índice de metacognição inclui cinco domínios: iniciativa, memória operacional, planejamento/organização, organização do material e monitoramento.

Os questionários dos pais, professores e versão pessoal levam de 10 a 15 minutos, cada um, para serem respondidos. Idealmente, os questionários devem ser preenchidos num ambiente calmo. Os escores dos questionários são expressos em escores padrão e ranks de percentis. Todas as medidas do BRIEF são convertidas em escore $T$, permitindo comparar os resultados do sujeito em relação ao grupo normativo.

A escala BRIEF foi normatizada na população americana em crianças e adolescentes entre 5 e 18 anos, ou seja, incluindo tanto a versão dos pais quanto a versão dos professores e a versão pessoal. Na versão pais e professores a amostra foi de 1.419 pais de 815 meninas e 604 meninos; 720 professores de 403 meninas e 317 meninos e para a versão pessoal 1.000 adolescentes, 448 meninos e 552 meninas, responderam ao questionário, respeitando as representatividades geográficas, étnica e social da população. O resultado da análise multivariada das variâncias revelou que idade e gênero são fatores significativos em vários domínios. A diferença significativa entre os resultados dos meninos e das meninas foi consistente tanto na versão dos pais quanto na versão dos professores justificando a necessidade de normas específicas segundo idade e gênero.

Baron (2000) realizou uma extensa revisão do BRIEF, comprovando suas sólidas propriedades psicométricas e promessa clínica. $\mathrm{O}$ autor citou estudos preliminares com subgrupos clínicos e mostrando que o BRIEF tem utilidade clínica em distinguir subgrupos diagnósticos, incluindo Transtorno do Déficit de Atenção com Hiperatividade (TDAH) e Transtornos Invasivos do Desenvolvimento (TID).

Uma edição especial do Child Neuropsychology (Donders, 2002) examinou o potencial do BRIEF na avaliação neuropsicológica pediátrica como uma medida de uma série de condições clínicas, incluindo autismo, transtorno bipolar, traumatismo crânio encefálico. Os resultados apresentados revelaram significativa elevação global no grupo clínico e diferença no perfil da elevação entre os grupos clínicos, dando suporte ao uso clínico do BRIEF.

A literatura internacional recomenda um cuidadoso processo de tradução e adaptação de testes (Noronha et al., 2003; Radanovic \& Mansur, 2002). Diferenças na origem e estrutura das línguas portuguesa e inglesa demandam um trabalho em profundidade para manter a qualidade do instrumento durante o processo de tradução e adaptação, garantindo a equivalência semântica e cultural com a versão original.

Beaton, Bombardier, Guillemin e Ferraz (2000) oferecem diretrizes que servem como um modelo para o processo de tradução e adaptação cultural. O processo envolve a adaptação de itens individuais, as instruções para os questionários, e as opções de resposta. Na primeira fase de adaptação é a tradução da língua original para a língua alvo. Os autores recomendam que pelo menos duas traduções sejam feitas por diferentes tradutores. Assim, as traduções podem ser comparadas e formulações mais pobres são identificadas e resolvidas em uma discussão entre os tradutores. Os dois tradutores devem ter diferentes perfis. Na próxima fase, os dois tradutores e um observador sintetizam os resultados da tradução. A partir da versão sintetizada das traduções, um tradutor totalmente cego para a versão original, traduz o questionário para o idioma original. Este é um processo de verificação de validade para se certificar de que a versão traduzida está refletindo o mesmo conteúdo da versão original.

A próxima fase é a composição do Comitê de Especialistas. Esta composição é fundamental para obter-se a equivalência cultural. O papel do comitê de especialistas é consolidar todas as versões do questionário e desenvolver o que seria considerado a versão pré-final do questionário para testes de campo. A comissão irá analisar, assim, todas as traduções e chegar a um consenso sobre qualquer discrepância. O material à disposição da comissão inclui o questionário original e cada tradução juntamente com os correspondentes relatórios escritos. Consenso deverá ser alcançado nos itens e, se necessário, a tradução e retrotradução devem ser repetidas.

O estágio final do processo de tradução seria o pré teste. Beaton et al. (2000) estabelece que, idealmente, 30 a 40 sujeitos devem ser testados. Cada sujeito é entrevistado sobre o que ele ou ela entendeu por cada item do questionário. A etapa final no processo de adaptação é uma apresentação de todos os relatórios e formulários para o autor do instrumento para se manter a par da versão traduzida.

Diante disso, o objetivo deste estudo foi traduzir, adaptar e avaliar a aplicabilidade da versão para o português do BRIEF, que será aqui denominado BREVE INVENTÁRIO DAS FUNÇÕES EXECUTIVAS (BRIFE), cujo procedimento assegura a obtenção das equivalências conceitual e semântica. 
Carim, D. B., Miranda, M. C. \& Bueno, O. F. A. (2012). Tradução e Adaptação para o Português do "Behavior Rating Inventory of Executive Function".

\section{Método}

\section{Fases na Tradução e Adaptação}

Etapa 1. Os questionários (Pais, Professores e Pessoal) foram traduzidos do inglês para o português, por professor de inglês, brasileiro, com conhecimento em psicologia, sendo, posteriormente, feita a retro tradução por outro profissional com conhecimentos da língua portuguesa e inglesa. A retro tradução ocorreu de modo independente, além de cega com relação ao perfil dos profissionais da primeira etapa. Em seguida, foi realizada revisão pelos editores (Psychological Assessment Resources).

Etapa 2. Primeiro Estudo-piloto. Essa primeira versão dos questionários foi respondida por pais e professores de 114 crianças e adolescentes (49 meninos e 65 meninas) entre 5 e 17 anos de idade no Estado do Rio de Janeiro. A versão pessoal foi respondida por 62 adolescentes entre 11 e 17 anos (25 meninos e 37 meninas). Os autores e colaboradores se reuniram com os aplicadores para discutir as dificuldades encontradas em cada item quanto ao nível de entendimento, tendo sido realizada a avaliação qualitativa: do valor funcional (medir o que se propõe, se atingiu o efeito-teto em alguma faixa etária) e da validade técnica (aplicabilidade) das perguntas.

Etapa 3. Foi realizada revisão da tradução e retro tradução por um comitê de juízes, formado por juízes bilíngües, sendo dois especialistas na área da neuropsicologia e em particular estudiosos em função executiva, e um leigo no assunto. Duas categorias de equivalência semântica entre os itens foram avaliadas por esses profissionais: significado referencial, que corresponde à concordância em termos de tradução literal entre um item original e o mesmo retraduzido e, significado geral, correspondente à concordância mais ampla, em termos de articulação de idéias e impacto entre um item original e sua retro tradução. Os autores do presente estudo avaliaram as apreciações feitas pelos especialistas, discutindo os itens e decidindo por aqueles que comporiam a versão brasileira.

Etapa 4. As novas versões dos questionários (Pais, Professores e Pessoal) foram aplicadas no segundo estudo piloto seguindo os mesmos procedimentos do primeiro estudo piloto, numa amostra constituída por 277 pais e 282 professores de crianças e adolescentes entre 5 e 18 anos de idade, e o questionário para adolescentes por 112 participantes entre 11 e 18 anos da Cidade do Rio de Janeiro (ver seção Amostra para detalhamento). Assim como no $1^{\circ}$ estudo-piloto, houve uma avaliação com base nos comentários críticos feitos pelos examinadores e nas suas anotações sobre os comportamentos. Além disso, foram realizadas análises estatísticas a fim de verificar as propriedades psicométricas preliminares da tradução e adaptação do BRIFE. Alguns itens das versões finais dos instrumentos (Pais, Professores, Pessoal) são apresentados no Anexo A (devido a direitos autorais não será apresentada a versão completa).

\section{Amostra}

Os participantes foram recrutados de escolas públicas e particulares, do Estado do Rio de Janeiro, e selecionados seguindo os critérios de exclusão: presença de doença neurológica e/ou psiquiátrica; repetição escolar; apresentar queixa no comportamento e/ou aprendizado; fazer uso de medicamento que possa interferir com as funções cognitivas/comportamentais, ter menos de 5 anos de idade ou mais de 18 anos de idade. E, seguindo os critérios de inclusão: ter pais e/ou cuidador responsáveis morando com a criança; déficit sensorial e/ou auditivo corrigido; o professor conhecer a criança/adolescente por pelo menos três meses. Para tanto, os professores preencheram o questionário Conners abreviado (Brito, 1987), adaptado para o contexto brasileiro, o qual é utilizado para screening de problemas de atenção, hiperatividade. Aquelas crianças que apresentavam escore acima do ponto de corte para idade e sexo não foram incluídas. Após esse processo inicial foi enviada uma carta de informação aos pais e o termo de consentimento livre e esclarecido para ser assinado. Após autorização dos pais, foi realizada a aplicação da versão abreviada da Escala de Inteligência Wechsler para Crianças (WISC-III; Wechsler, 2002) para os participantes entre 6 e 16 anos, e a Escala de Inteligência Wechsler para Adultos (WAIS-III; Wechsler, 1997) entre 17 e 18 anos, ou seja, a aplicação dos subtestes cubos e vocabulário, que permitem uma estimativa da capacidade cognitiva global, através do QI estimado, já que dados da literatura mostram altas correlações entre as formas reduzidas (QI estimado) e as formas completas (QI completo; Mello et al., 2011). Aquelas crianças e adolescentes que apresentavam resultado abaixo do esperado não foram incluídas na amostra (QI estimado <80). Todos os procedimentos do presente estudo foram aprovados pelo Comitê de Ética em Pesquisa da Universidade Federal de São Paulo - Escola Paulista de Medicina (CEP 16741/07).

A amostra final nos três questionários do BRIFE (Pais, Professores e Pessoal) foi obtida através do recrutamento das escolas públicas e particulares no cenário urbano e suburbano no Estado do Rio de Janeiro, por conveniência dada a dificuldade de aceitação por parte das escolas. O questionário para pais foi respondido por um total de 277 pais, sendo 170 para a faixa entre 5 e 11 anos, e 107 para a faixa entre 11 e 18 anos de idade (média de 9,87 anos e um desvio padrão de 3,41), com 165 dos participantes sendo do sexo feminino $(59,6 \%)$. Já o questionário para professores, a amostra foi constituída por 282 professores, sendo 180 professores de alunos na faixa etária 5-11 anos e 102 da faixa 11-18 anos, (média de 9,70 anos e um desvio padrão de 3,28), e 162 participantes do sexo feminino (57,4\%). Na forma pessoal, a amostra foi de 112 respondentes, sendo 71 do sexo feminino $(63,4 \%)$ e com idade entre 11 e 18 anos, média de 13,58 anos e um desvio padrão de 1,97 , conforme a Tabela 1 . 
Psicologia: Reflexão e Crítica, 25(4), 653-661.

Tabela 1

Distribuição dos Sujeitos em Função do Sexo e da Idade

\begin{tabular}{|c|c|c|c|c|c|c|c|c|c|c|c|c|c|}
\hline \multirow{3}{*}{$\begin{array}{l}\text { Faixa Etária } \\
\text { (em anos) }\end{array}$} & \multicolumn{4}{|c|}{ Versão Pais } & \multicolumn{4}{|c|}{ Versão professor } & \multicolumn{4}{|c|}{ Versão Pessoal } & \multirow{3}{*}{$\begin{array}{l}\text { Tota } \\
\quad N\end{array}$} \\
\hline & \multicolumn{2}{|c|}{ Feminino } & \multicolumn{2}{|c|}{ Masculino } & \multicolumn{2}{|c|}{ Feminino } & \multicolumn{2}{|c|}{ Masculino } & \multicolumn{2}{|c|}{ Feminino } & \multicolumn{2}{|c|}{ Masculino } & \\
\hline & $N$ & $\%$ & $N$ & $\%$ & $N$ & $\%$ & $N$ & $\%$ & $N$ & $\%$ & $N$ & $\%$ & \\
\hline 5 a 6 & 32 & 19,4 & 23 & 20,5 & 33 & 20,4 & 23 & 19,2 & - & - & - & - & 111 \\
\hline 7 a 8 & 24 & 14,5 & 26 & 23,2 & 26 & 16,0 & 28 & 23,3 & - & - & - & - & 104 \\
\hline 9 a 10 & 41 & 24,9 & 24 & 21,4 & 43 & 26,5 & 27 & 22,5 & - & - & - & - & 135 \\
\hline 11 a 12 & 21 & 12,7 & 16 & 14,3 & 23 & 14,2 & 18 & 15,0 & 21 & 29,6 & 15 & 36,6 & 114 \\
\hline 13 a 14 & 29 & 17,6 & 12 & 10,7 & 22 & 13,6 & 13 & 10,8 & 29 & 40,8 & 13 & 31,7 & 118 \\
\hline $15 \mathrm{a} 18$ & 18 & 10,9 & 11 & 9,8 & 15 & 9,3 & 11 & 9,2 & 21 & 29,6 & 13 & 31,7 & 89 \\
\hline Total & 165 & 100,0 & 112 & 100,0 & 162 & 100,0 & 120 & 100,0 & 71 & 100,0 & 41 & 100,0 & 671 \\
\hline
\end{tabular}

\section{Procedimentos}

Todas as análises foram realizadas a partir dos escores brutos dos questionários. Como citado anteriormente, cada questão do questionário é respondida como "nunca" (escore 0), "algumas vezes" (escore 1) e "constantemente" (escore 2). Para a análise psicométrica dos itens, investigou-se o parâmetro de discriminação com base no cálculo da correlação item-total $\left(\mathrm{r}_{\mathrm{it}} ;\right.$ Erthal, 1987). Considerou-se o item discriminativo quando a correlação entre os itens foi maior ou igual a 0,25 , o que indica que o item contribui para diferençar as pessoas em relação ao que foi medido. Foram calculadas as correlações item-total de cada afirmação e o total da escala a que pertenciam nas três versões do BRIFE (Pais, Professores e Pessoal).

Para analisar a consistência interna dos questionários foi calculado o coeficiente Alfa de Cronbach. Seguindo as orientações do manual original do teste, foram realizadas análises fatoriais exploratórias com as escalas do BRIFE. Os cálculos foram feitos com o total de pontos (escore bruto) de cada domínio dos questionários Pais, Professores e Pessoal.

O método de extração utilizado foi a Análise de Componentes Principais, rotação Direct Oblimin com normalização Kaiser. Foram extraídos dois componentes utilizando como critério Eigenvalues maiores do que 1 . O critério adotado no manual original do BRIEF são cargas fatoriais acima de 0,40, mantido como carga mínima para manter o item.

No questionário Pessoal, o método de extração utilizado foi o Principal Axis Factoring (PAF). Utilizando o critério de Eigenvalue maior do que 1 foi extraído apenas um componente. Uma vez que como nos casos dos questionários dos Pais e Professores era esperado que fossem extraídos dois componentes, foi rodada uma nova análise fatorial utilizando o método PAF com a rotação Direct Oblimin com normalização Kaiser, solicitando a extração de dois componentes.

\section{Resultados}

O resultados das análises qualitativas das etapas 1 e 2 que abrangeu o processo inicial de tradução, retro tradução e as sugestões de correções feitas pela editora.
Houve as seguintes principais modificações sugeridas no processo de retro tradução:

1. O termo "overwhelmed" (questionário Pessoal: item 75; questionário dos Professores: item 37) foi traduzido como "oprimidos". Ao termo 'overwhelmed', a versão original pressupõe uma sensação de sentirse como uma tarefa ou atribuição "demais para suportar", de modo que a pessoa sente que não pode iniciar ou abordar a tarefa. Assim, a versão do questionário Pessoal ficou: "Facilmente me sinto sobrecarregado como se tivesse muita coisa para fazer"; a forma do questionário dos professores ficou: "Sente-se sobrecarregado com deveres escolares longos".

2. Em alguns casos, a utilização do termo "large assignment" (questionário dos Professores: item 37; questionário dos Pais: item 36), traduzido inicialmente como: "avaliações longas", deveria indicar uma tarefa de casa, algo que se estuda. Fazer uma avaliação na escola nos Estados Unidos tem um tempo limitado. Assim, foi modificado para: "deveres escolares longos" e "exercícios longos", respectivamente.

$\mathrm{Na}$ análise qualitativa do primeiro estudo piloto, verificou-se dúvidas dos respondentes (Pais, Professores e Pessoal) nas questões de dupla negação, ou seja, nas questões que começam com "Não". Portanto, as questões foram reformuladas para começarem com afirmativas (por ex. "não percebe quando seu comportamento causa uma reação negativa" foi alterada para "tem dificuldade para perceber quando seu comportamento causa uma reação negativa"). A análise qualitativa mostrou que houve melhor compreensão por parte dos respondentes no segundo estudo piloto, independente da sua escolaridade.

Após esses resultados preliminares dessa primeira versão do BRIFE, houve a análise do comitê de juízes o qual avaliou a tradução, retro tradução e a adaptação, assim como também a equivalência semântica e a atribuição de notas [quanto ao grau de modificação: inalterado (0), pouco alterado (1), muito alterado (2) e completamente alterado (3)]. Os juízes sugeriram algumas modificações, por ex. "deve-se evitar voz passiva em questionário". Assim, o item 2 da forma para pais que inicialmente fora 
Carim, D. B., Miranda, M. C. \& Bueno, O. F. A. (2012). Tradução e Adaptação para o Português do "Behavior Rating Inventory of Executive Function".

traduzido por "Quando são dadas três coisas para fazer, lembra somente da primeira ou da última" foi modificado para: "quando lhe dão três coisas para fazer, lembra somente da primeira ou da última".
No que se refere aos resultados das Análises Psicométricas das Escalas do BRIFE, As Tabelas 2 e 3 mostram as médias e desvios-padrão de cada versão (Pais, Professores e Pessoal) do BRIFE.

Tabela 2

Média e DP do Escore Bruto do BRIFE em Função da Faixa Etária do Questionário para Pais e Professores

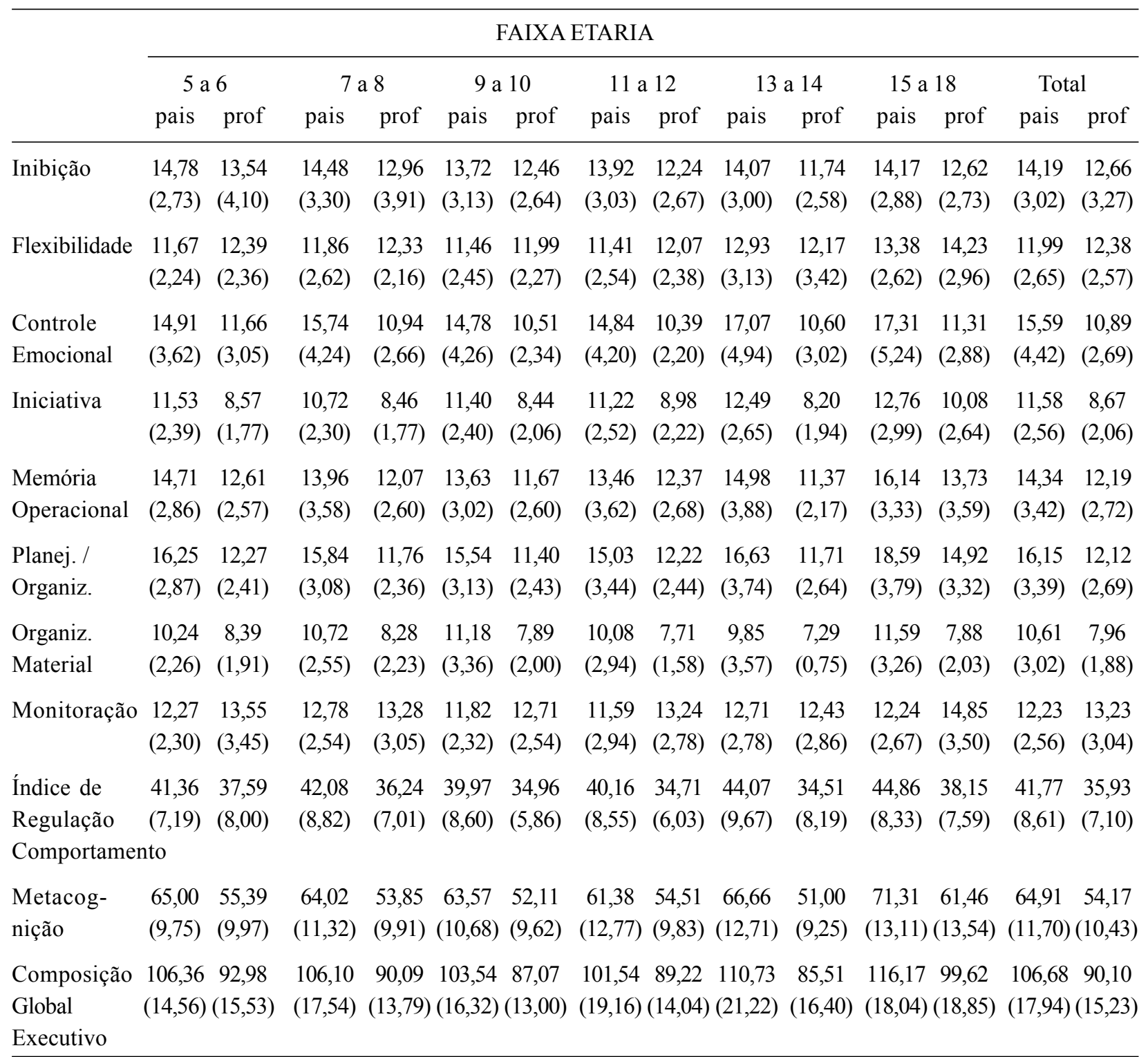

Nota. Obs: prof: professores.

A Análise dos Itens, no questionário para pais, as Correlações Item-Total mostraram que das 72 correlações calculadas para os itens que compõem o teste, apenas 3 itens $(4,17 \%)$ foram inferiores a 0,25 indicando que estes itens foram pouco discriminativos: o item 8 da escala Flexibilidade $(0,221)$; o item 31 da escala Monitoração $(0,226)$ e o item 66 da escala Iniciativa $(0,248)$.

No questionário para professores todas as correlações foram superiores a 0,25 . E no questionário pessoal das 80 correlações calculadas, apenas 3 itens foram inferiores a 0,25 evidenciando itens pouco discriminativos: item 59 da escala Organização de Materiais (0,108); item 34 da escala Monitoramento $(0,212)$ e item 46 da escala Inibição $(0,183)$.

Quanto a analise de confiabilidade, a Tabela 4 mostra os valores dos Alfas de Cronbach, sendo que no questionário dos Pais os índices oscilaram entre 0,671 (Monitoração) e 0,936 (Composição Global Executivo). Já no questionário dos Professores oscilaram entre 0,784 (Monitoração) e 0,951 (Composição Global Executivo),e para o questionário Pessoal oscilaram entre 0,743 (Monitoração) e 0,957 (Composição Global Executivo), indicando assim adequados coeficientes de fidedignidade do teste. 
Tabela 3

Média e DP do Escore Bruto do BRIFE em Função da Faixa Etária do Questionário Pessoal

\begin{tabular}{lcccc}
\hline & \multicolumn{4}{c}{ Faixa Etária } \\
\cline { 2 - 5 } & 11 a 12 & 13 a 14 & 15 a 18 & Total \\
\hline Inibição & $18,81(4,12)$ & $22,10(5,58)$ & $21,21(3,91)$ & $20,77(4,83)$ \\
Flexibilidade & $14,47(3,02)$ & $15,67(3,82)$ & $16,18(3,32)$ & $15,44(3,47)$ \\
Flexibilidade Comportamento & $7,19(1,64)$ & $7,90(2,31)$ & $8,29(1,96)$ & $7,79(2,04)$ \\
Flexibilidade Cognitiva & $7,28(1,80)$ & $7,76(2,07)$ & $7,88(2,07)$ & $7,64(1,98)$ \\
Controle Emocional & $15,97(3,87)$ & $17,55(4,44)$ & $17,97(4,52)$ & $17,17(4,33)$ \\
Completar Tarefas & $13,50(3,23)$ & $15,55(3,86)$ & $15,71(3,50)$ & $14,94(3,67)$ \\
Memória Operacional / trabalho & $17,92(3,94)$ & $19,81(4,67)$ & $20,24(4,16)$ & $19,33(4,37)$ \\
Planejamento /Organização & $17,67(3,89)$ & $19,79(5,12)$ & $20,26(4,30)$ & $19,25(4,60)$ \\
Organização do Material & $9,42(2,10)$ & $10,76(3,32)$ & $11,15(3,11)$ & $10,45(2,98)$ \\
Monitoração & $7,33(2,15)$ & $8,31(2,22)$ & $7,82(2,17)$ & $7,85(2,20)$ \\
Índice de Regulação do Comportamento & $56,58(11,42)$ & $63,62(13,78)$ & $63,18(11,17)$ & $61,22(12,60)$ \\
Metacoginição & $58,50(11,36)$ & $65,90(15,01)$ & $67,35(13,05)$ & $63,96(13,75)$ \\
Composição Global Executivo & $115,08(20,70)$ & $129,52(26,87)$ & $130,53(21,73)$ & $125,19(24,31)$ \\
\end{tabular}

Tabela 4

Valores do Alfa de Cronbach - Questionário Pais, Professores, Pessoal

\begin{tabular}{|c|c|c|c|c|c|c|}
\hline \multirow[t]{2}{*}{ Índices } & \multicolumn{2}{|c|}{$\begin{array}{l}\text { Questionário } \\
\text { Pais }\end{array}$} & \multicolumn{2}{|c|}{$\begin{array}{c}\text { Questionário } \\
\text { Professores }\end{array}$} & \multicolumn{2}{|c|}{$\begin{array}{c}\text { Questionário } \\
\text { Pessoal }\end{array}$} \\
\hline & Itens & $\begin{array}{c}\text { Alfa de } \\
\text { Cronbach }\end{array}$ & Itens & $\begin{array}{l}\text { Alfa de } \\
\text { Cronbach }\end{array}$ & Itens & $\begin{array}{r}\text { Alfa de } \\
\text { Cronbach }\end{array}$ \\
\hline Inibição & 10 & 0,751 & 10 & 0,872 & 13 & 0,834 \\
\hline Flexibilidade & 8 & 0,703 & 10 & 0,784 & 10 & 0,763 \\
\hline Flexibilidade do Comportamento & - & - & - & - & 10 & 0,822 \\
\hline Flexibilidade Cognitiva. & - & - & - & - & 10 & 0,822 \\
\hline Controle Emocional & 10 & 0,882 & 9 & 0,869 & 12 & 0,805 \\
\hline Completar Tarefas & - & - & - & - & 13 & 0,826 \\
\hline Iniciativa & 8 & 0,675 & 7 & 0,787 & & \\
\hline Memória Operacional & 10 & 0,814 & 10 & 0,841 & 7 & 0,784 \\
\hline Planejamento/Organização & 12 & 0,768 & 10 & 0,837 & 5 & 0,743 \\
\hline Organização do Material & 6 & 0,790 & 7 & 0,841 & 5 & 0,661 \\
\hline Monitoração & 8 & 0,671 & 10 & 0,819 & 5 & 0,677 \\
\hline Índice Regulação do Comportamento & 28 & 0,901 & 29 & 0,915 & 38 & 0,924 \\
\hline Metacognição & 44 & 0,911 & 44 & 0,944 & 42 & 0,935 \\
\hline Composição Global Executivo & 72 & 0,936 & 73 & 0,951 & 80 & 0,957 \\
\hline
\end{tabular}

Nota. Obs: Itens - refere-se á quantidade de questões que compõe o índice.

Por fim, a Tabela 5 mostra os resultados da análise fatorial realizada com o total de pontos (escore bruto) de cada domínio dos questionários dos Pais e Professores. Foram extraídos dois componentes quando se utilizou como critério os valores de Eigenvalue maiores do que 1. Ao refazer os cálculos solicitando a extração direta de dois componentes (sem o critério do Eigenvalue) os resultados foram os mesmos.

No questionário dos Pais, os fatores ficaram claramente diferenciados para as escalas que compõem a Metacognição (Fator 1) e o Índice de Regulação do Comportamento
(Fator 2). Quando são comparados estes resultados com os da amostra normativa do manual do teste na população americana, percebe-se que nos estudos brasileiros os fatores aparecem definidos de forma mais adequada uma vez que no estudo original a escala Inibição não apresentou carga no fator Índice de Regulação do Comportamento (como era esperado), mas sim na Metacognição.

No questionário dos Professores, pode-se verificar que ainda que as escalas se agruparam adequadamente em dois fatores, a escala Monitoração apresentou carga fatorial principal no Fator 1 junto com as outras escalas que 
Carim, D. B., Miranda, M. C. \& Bueno, O. F. A. (2012). Tradução e Adaptação para o Português do "Behavior Rating Inventory of Executive Function".

Tabela 5

Cargas Fatoriais Piloto 2 - Questionário dos Pais e Professores

\begin{tabular}{|c|c|c|c|c|}
\hline \multirow[b]{2}{*}{ Escalas } & \multicolumn{2}{|c|}{ Pais } & \multicolumn{2}{|c|}{ Professores } \\
\hline & Fator 1 & Fator 2 & Fator 1 & Fator 2 \\
\hline Inibição & & 0,608 & 0,753 & 0,685 \\
\hline Flexibilidade & & 0,467 & 0,763 & 0,559 \\
\hline Controle Emocional & & 0,977 & 0,680 & 0,987 \\
\hline Iniciativa & 0,686 & & 0,722 & \\
\hline Memória Operacional / trabalho & 0,895 & & 0,834 & \\
\hline Planejamento/Organização & 0,908 & & 0,826 & \\
\hline Organização do Material & 0,443 & & 0,689 & \\
\hline Monitoração & 0,598 & & 0,806 & 0,487 \\
\hline \% de Variância explicada & \multicolumn{2}{|c|}{57,03} & \multicolumn{2}{|c|}{65,55} \\
\hline KMO & \multicolumn{2}{|c|}{0,836} & \multicolumn{2}{|c|}{0,782} \\
\hline Teste de Esfericidade de Bartlett & \multicolumn{2}{|c|}{1009,800} & \multicolumn{2}{|c|}{1494,656} \\
\hline Correlações entre os fatores & \multicolumn{2}{|c|}{0,56} & \multicolumn{2}{|c|}{0,400} \\
\hline
\end{tabular}

Nota. Obs: o critério adotado no manual original do teste são cargas fatoriais acima de 0,40.

Tabela 6

Cargas Fatoriais Piloto 2 Questionário Pessoal

\begin{tabular}{lcc}
\hline Escalas & Fator 1 & Fator 2 \\
\hline Inibição & & 0,532 \\
Flexibilidade & & 0,715 \\
Controle Emocional & 0,302 & 0,459 \\
Monitoração & 0,904 & \\
Memória Operacional / trabalho & 0,857 & \\
Planejamento /Organização & 0,730 & \\
Organização do Material & 0,782 & \\
Completar Tarefas & $66,49 \%$ & \\
\% de Variância explicada & 0,890 & \\
KMO & 576,362 & \\
Teste de Esfericidade de Bartlett & 0,70 & \\
Correlações entre os fatores & 0,0 \\
\hline
\end{tabular}

Nota. Obs: o critério adotado no manual original do teste são cargas fatoriais acima de 0,40 .

compõe a Metacognição (como era esperado teoricamente) e uma carga secundária no Fator 2 que é formado pelas escalas que constituem o Índice de Regulação do Comportamento.

A Tabela 6 mostra os resultados para o questionário Pessoal, considerando o método de extração principal Axis Factoring (PAF). Utilizando o critério de Eigenvalue maior do que 1 foi extraído apenas um componente. Uma vez que como nos casos dos questionários dos Pais e Professores era esperado que fossem extraídos dois componentes, foi rodada uma nova análise fatorial utilizando o método PAF com a rotação Direct Oblimin com normalização Kaiser, solicitando a extração de dois fatores. As escalas foram agrupadas de forma a compor os dois fatores que apresentam justificativa teórica, ou seja, o Fator 1 Metacognição e Fator 2 Índice de Regulação do Comportamento com exceção do fato que a escala Monitoração que apesar de ter a carga principal no fator 2 (ao qual teoricamente ela pertence) apresenta também uma carga secundária no fator 1 .

\section{Discussão e Conclusões}

O presente estudo traduziu e adaptou o Behavior Rating Inventory of Executive Functions (BRIEF) aqui denominado BREVE INVENTÁRIO DAS FUNÇÕES EXECUTIVAS (BRIFE). Há uma lacuna de instrumentos, em língua portuguesa do Brasil, com essas características, ou seja, que avalia o funcionamento executivo na vida diária da criança e do adolescente. O instrumento é amplamente utilizado em diversos estudos clínicos assim como há propriedades psicométricas adequadas na sua versão original em inglês.

As três versões do BRIFE (pais, professores e pessoal) tiveram o mesmo processo de tradução e adaptação de testes recomendado na literatura internacional, que garantem a equivalência semântica e cultural com a versão original. Nesse caso, o instrumento deve ser capaz de captar adequadamente o conceito subjacente das funções executivas, assim como mostrar-se capaz de obter na cultura para a qual está sendo adaptado o mesmo efeito que o instrumento original tem no contexto em que foi criado. Para se obter a versão em português, válida e confiável, foi necessário percorrer uma série de etapas e a avaliação semântica foi uma delas (possibilitando estudar o conceito corretamente). Deve-se enfatizar que o processo de tradução e adaptação transcultural deve ter uma base conceitual e apoiar-se em metodologias criteriosamente definidas. Apesar de ser trabalhoso e dispendioso esse processo pro- 
porciona benefícios indiscutíveis ao gerar um instrumento que permite obter informações, de fato, válidas. No presente estudo, após um longo processo de tradução e adaptação, os resultados parecem apontar para uma boa aplicação e entendimento do instrumento na amostra investigada.

A análise qualitativa e julgamento dos especialistas mostraram-se fundamentais visto que algumas questões apresentaram, na tradução, dupla negação, como por ex. "is not a self-starter" foi traduzido para "não tem iniciativa". Desta forma, houve dúvida entre os respondentes em assinalar: "nunca", "algumas vezes" ou "freqüentemente". Esta etapa também se mostrou fundamental para verificar que o instrumento apresenta uma linguagem clara e acessível a qualquer respondente.

A análise estatística preliminar permitiu dados de verificação das propriedades psicométricas iniciais da tradução e adaptação do BRIEF como recomendado por diversos autores (Beaton et al., 2000; Hambleton \& Bollwark, 1991).

Optou-se, nesse estudo, não analisar efeitos de gênero, idade, e diferenças entre escola pública e escola privada mesmo que preliminarmente, pois a distribuição não foi totalmente homogênea, de tal forma que permitisse dados consistentes em relação a esses efeitos. De qualquer forma, é o processo de padronização, com melhor estratificação da população em estudo que permitirá tais análises.

Ainda assim, o número de sujeitos, muito acima do recomendado na literatura para estudo piloto de tradução e adaptação de instrumento, a saber 30-40 sujeitos segundo Beaton et al. (2000) permitiu uma análise das propriedades psicométricas iniciais do instrumento. $\mathrm{Na}$ análise da equivalência de itens e semântica, optou-se por permanecer com todos os itens dos questionários na versão em português ( 86 itens no questionário dos pais; 86 itens no questionário dos professores; 80 itens no questionário pessoal), conservando os poucos que mostraram menos consistência, porque mesmo havendo menos consistência a equivalência foi muito importante para a adaptação do instrumento como um todo.

Os escores de Alfa de Cronbach encontrados na versão brasileira se assemelham aos relatados na versão original, indicando boa consistência interna do instrumento nas três versões do BRIFE.

No questionário dos pais do segundo estudo piloto, as análises fatoriais quando comparados com os resultados da amostra normativa do manual do teste, os fatores aparecem definidos de forma mais adequada uma vez que no estudo original a escala Inibição não apresentou carga no fator Índice de Regulação do Comportamento (como era esperado), mas sim na Metacognição.

No questionário dos professores do segundo estudo piloto, ainda que as escalas tenham se agrupado adequadamente em dois fatores, a escala Monitoração apresentou carga fatorial principal no Fator 1 junto com as outras escalas que compõem a Metacognição (como era esperado teoricamente) e uma carga secundária no Fator 2 que é formado pelas escalas que constituem o Índice de Regu- lação do Comportamento. Na versão original, a escala Monitoração apresentou uma carga secundária no Fator 2. Como a carga fatorial maior ocorreu no fator correto isso pode não ser um problema.

No questionário pessoal do segundo estudo piloto, utilizando o Método de Extração Principal Axis FActoring (PAF) e o critério de Eigenvalue maiores do que 1 foi extraído apenas um fator. Como nos casos dos questionários dos Pais e Professores era esperado que fossem extraídos dois fatores (Fator 1: Metacognição; Fator 2: Inibição do Comportamento), foi rodada uma nova análise fatorial e o resultado foi o agrupamento das escalas de forma a compor os dois fatores que apresentam justificativa teórica, ou seja, Fator 1 Metacognição e Fator 2 o Índice de Regulação do Comportamento com exceção do fato que a escala Monitoração que apesar de ter a carga principal no Fator 2 (ao qual ela teoricamente pertence) apresenta também uma carga secundária no fator 1. Em nosso estudo, esta diferença pode ter sido devida a uma variação amostral.

\section{Considerações Finais}

O presente estudo disponibiliza para o português um instrumento de avaliação do desempenho das funções executivas no dia-a-dia da criança e/ou adolescente. A importância deste trabalho deve-se ao fato de ser o primeiro documento na população brasileira com esta finalidade. Diferenças na origem e estrutura das línguas portuguesa e inglesa demandaram um trabalho em profundidade para manter a qualidade do instrumento durante o processo de tradução e adaptação, garantindo a equivalência semântica e cultural com a versão original. $O$ BRIFE se mostrou uma escala de fácil aplicação e compreensão pela população alvo. Sua adequada confiabilidade sugere ser este um instrumento complementar importante de avaliação na prática do neuropsicólogo clínico.

O presente estudo apresenta algumas limitações. Primeiramente o número de sujeitos por idade foi baixo, o que nos levou a agrupá-los por faixa etária, a cada 2 anos entre os 5 e 14 anos, e a partir disso agrupando em única faixa os participantes de 15 a 18 anos de idade, o que pode ter tido influência nas análises fatoriais preliminares aqui realizadas. Outra limitação diz respeito ao fato de que não foram analisados, mesmo que preliminarmente, possíveis efeitos de gênero, idade já que a normatização americana mostrou efeitos dessas variáveis. Mas também, considera-se relevante a análise de tipo de escola, que pode testar hipóteses da influência do nível sócio-econômico e ainda de fatores culturais, considerando amostras de diferentes regiões do nosso país, como tem sido apontado em diversos estudos em nossa população. Sendo assim, futuros estudos de normatização do instrumento para a população brasileira poderão contribuir não só para analise dessas variáveis, mas também para melhor refinamento do instrumento, obtendo-se evidências de fidedignidade, 
validade de construto e de critério, além de perfis neuropsicológicos clínicos de crianças com quadros neurológicos e/ou psiquiátricos.

De qualquer forma, nosso estudo mostra que, como afirmam Mattos et al. (2006), o uso de uma sistemática mais detalhada para a tradução e a adaptação transcultural de uma escala é fundamental para que se possam localizar as dificuldades de sua equivalência semântica, mostrando-se um método mais adequado que a simples tradução-retro tradução do instrumento.

\section{Referências}

Baron, I. S. (2000). Test Review: Behavior Rating Inventory of Executive Function. Child Neuropsychology, 6, 235-238.

Beaton, D. E., Bombardier, C., Guillemin, F., \& Ferraz, M. B. (2000). Guidelines for the process of cross-cultural adaptation of self-report measures. Spine, 25(4), 3186-3191.

Bodnar, L. E., Prahme, M. C., Cutting, L. E., Denckla, M. B., \& Mahone, E. M. (2007). Construct validity of parent rating of inhibitory control. Child Neuropsychology, 13, 345-362.

Brito, G. N. (1987). The Conners Abbreviated Teaching Rating Scale: Development of norms in Brazil. Journal of Abnormal Child Psychology, 15(4), 511-518.

Denckla, M. B. (1994). Measurement of executive function. In G. R. Lyon (Ed.), Frames of reference for the assessment of learning disabilities: New views on measurement issues (pp. 117-142). Baltimore, MD: Paul Brookes.

Donders, J. (2002). The Behavior Rating Inventory of Executive Function: Introduction. Child Neuropsychology, 8(4), 229230.

Erthal, T. C. (1987). Manual de Psicometria. Rio de Janeiro, RJ: Zahar.

Gioia, G. A., Isquith, P. K., Guy, S. C., \& Kenworthy, L. (2000). Behavior Rating Inventory of Executive Function. Professional manual. Lutz, FL: Psychological Assessment Resource.

Gioia, G. A., Isquith, P. K., Kenworthy, L., \& Barton R. M. (2002). Profiles of everyday executive function in acquired and developmental disorders. Child Neuropsychology, 8(2), 121-137.

Hambleton, R. K., \& Bollwark, J. (1991). Adapting Tests for use in different cultures: Technical issues and methods. ITC Bulletin/Bulletin CIT: International Test Bulletin Issues, 32/ 33, 3-32.
Heaton, R. K., Chelune, G. J., Talley, J. L., Kay, G. G., \& Curtiss, G. (1993). Wisconsin Card Sorting Test Manual Revised and Expanded. Luts, FL: Psychological Assessment Resources.

Mattos, P., Segenreich, D., Saboya, E., Souza, M., Dias, G., \& Romano, M. (2006). Transcultural adaptation of the Adult Self-Report Scale into Portuguese for Evaluation of Adult Attention-Deficit/Hyperactivity Disorder (ADHD). Revista de Psiquiatria Clínica, 33(4), 188-194.

Mello, C. B., Argollo, N., Shayer, B. P. M., Abreu, N., Godinho, K., Durán, P., et al. (2011). Versão abreviada do WISC-III: Correlação entre QI estimado e QI total em crianças brasileiras. Psicologia: Teoria e Pesquisa, 27(2), 149-155.

Noronha, A. P. P., Vendramin, C. M. M., Cangurú, C., Souza, C. V. R., Cobêro, C., de Paula, L. M., et al. (2003). Propriedades psicométricas apresentadas em manuais de testes de inteligência. Psicologia em Estudo, 8(1), 93-99.

Radanovic, M., \& Mansur, L. L. (2002). Performance of a Brazilian population sample in the Boston Diagnostic Aphasia Examination. A pilot study. Brazilian Journal of Medical and Biological Research, 35, 305-317.

Slick, D. J., Lautzenhiser, A., Sherman, E. M. S., \& Eyrl, K. (2006). Frequency of Scale Elevations and Factor Structure of the Behavior Rating Inventory of Executive Function (BRIEF) in children and adolescent with intractable epilepsy. Child Neuropsychology, 12, 181-189.

Sullivan, J., \& Riccio, C. A. (2007). Diagnostic group differences in parent and teacher rating on the BRIEF and Conners' Scales. Journal of Attention Disorders, 11(3), 398-406.

Wechsler, D. (2002). WISC-III: Escala de Inteligência Wechsler para crianças (3. ed., V. L. M. Figueiredo, adaptação e padronização brasileira). São Paulo, SP: Casa do Psicólogo.

Wechsler, D. (1997). Wechsler Adult Intelligence Scale: Administration and scoring manual ( $3^{\text {rd }}$ ed.). San Antonio, TX: The Psychological Corporation.

Weyandt, L. L. (2005). Executive function in children, adolescents and adults with attention déficit hyperactivity disorder: Introduction to the Special Issue. Developmental Neuropsychology, 27(1), 1-10.

\section{$\operatorname{AnexoA}$}

Exemplos dos Itens da Versão Final do Breve Inventário das Funções Executivas (BRIFE)

\section{INIBIÇÃO}

- Interrompe os outros

- Perde a calma mais do que os amigos

- Fala coisas sem pensar

- Tem um comportamento muito agressivo ou perde o controle

FLEXIBILIDADE

\section{CONTROLE EMOCIONAL}

- Fica chateado com situações novas

- Fica irritado com mudanças de planos

- Fica perturbado com mudanças de professor ou de turma

- Tem dificuldades em aceitar situações novas (turma, grupos, amigos)
Recebido: 08/12/2010

$1^{a}$ revisão: 16/08/2011 Aceite final: 06/09/2011 